\title{
Existence of chaos for partial difference equations via tangent and cotangent functions
}

Haihong Guo ${ }^{1}$ and Wei Liang ${ }^{1 *}$

Correspondence:

wliang@hpu.edu.cn

'School of Mathematics and

Information Science, Henan

Polytechnic University, Jiaozuo

Henan, China

\begin{abstract}
This paper is concerned with the existence of chaos for a type of partial difference equations. We establish four chaotification schemes for partial difference equations with tangent and cotangent functions, in which the systems are shown to be chaotic in the sense of Li-Yorke or of both Li-Yorke and Devaney. For illustration, we provide three examples are provided.
\end{abstract}

Keywords: Chaos; Partial difference equation; Li-Yorke chaos; Devaney chaos

\section{Introduction}

In this paper, we focus on the existence of chaos in the following partial difference equation:

$$
x(n+1, m)=f(x(n, m), x(n, m+1)),
$$

where $n \geq 0$ is the time step, $m$ is the lattice point with $0 \leq m \leq k<+\infty, f: D \subset \mathbf{R}^{2} \rightarrow \mathbf{R}$ is a map, and $k+1$ is the system size. In many engineering applications, such as imaging, digital filter, and spatial dynamical system, Eq. (1) plays an important role [1, 2].

In the past years, with the development of chaos theory, chaos has been applied in many fields, such as physics, chemistry, engineering, and mathematics. In mathematics, chaos has become a significant branch of dynamical systems [3]. Furthermore, anticontrol of chaos (chaotification) is an important branch of chaos, and many researchers devoted much effort to chaotification. The first important result was obtained by Chen and Liu [4] proved that Eq. (1) in $\mathbf{R}^{3}$ is chaotic in the Li-Yorke sense by constructing spatial periodic orbits of specified period. Later, Eq. (1) was reformulated into a discrete system [5]. By applying this method Shi [6] established some criteria of chaos by applying chaos in scalar ordinary difference equations and snap-back repeller theory. Recently, chaotification problems for Eq. (1) with general controllers, sawtooth functions, and mod operations were studied, respectively, and all the controlled systems were proved to be chaotic in the sense of both Devaney and Li-Yorke [7-9]. In [10], two chaotification schemes of Eq. (1)

(c) The Author(s) 2020. This article is licensed under a Creative Commons Attribution 4.0 International License, which permits use, sharing, adaptation, distribution and reproduction in any medium or format, as long as you give appropriate credit to the original author(s) and the source, provide a link to the Creative Commons licence, and indicate if changes were made. The images or other third party material in this article are included in the article's Creative Commons licence, unless indicated otherwise in a credit line to the material. If material is not included in the article's Creative Commons licence and your intended use is not permitted by statutory regulation or exceeds the permitted use, you will need to obtain permission directly from the copyright holder. To view a copy of this licence, visit http://creativecommons.org/licenses/by/4.0/. 
via sine functions,

$$
x(n+1, m)=f(x(n, m), x(n, m+1))+\varepsilon \sin (\mu x(n, m)),
$$

were established for $\mu>1$. Furthermore, we proved that not only the above controlled system but also Eq. (1) with cosine functions are chaotic in the sense of both Li-Yorke and Devaney for $\mu=1$ [11].

As one of the main elements of basic elementary functions, trigonometric functions are of great importance. Sine, cosine, tangent, and cotangent functions are basic ones. It is known that sine and cosine are continuous and have a similar geometric shape with sawtooth functions and mod operations $[6-8,12,13]$. However, tangent and cotangent are piecewise continuous, and their geometric shapes are different from those of sine, cosine, sawtooth, and mod. Can tangent and cotangent functions be viewed as controllers to make the controlled Eq. (1) to be chaotic? In this paper, we attempt to address such an interesting question and try to establish chaotification schemes for the following controlled systems:

$$
\begin{aligned}
& x(n+1, m)=f(x(n, m), x(n, m+1))+\varepsilon \tan (x(n, m)), \\
& x(n+1, m)=f(x(n, m), x(n, m+1))+\varepsilon \cot (x(n, m)), \\
& x(n+1, m)=f(x(n, m), x(n, m+1))+\varepsilon \tan (x(n, m+1)), \\
& x(n+1, m)=f(x(n, m), x(n, m+1))+\varepsilon \cot (x(n, m+1)) .
\end{aligned}
$$

The rest of this paper is organized as follows. In Sect. 2, we list some basic concepts and lemmas about chaos. In Sects. 3, we consider anticontrol of chaos of Eq. (1) with tangent and cotangent functions, give four theorems, and prove that all the controlled systems are chaotic in the sense of Li-Yorke or of both Li-Yorke and Devaney by the coupledexpansion theory. Finally, in Sect. 4, we provide three illustrative examples.

\section{Preliminaries}

Now we introduce some basic concepts and lemmas.

Definition 1 ([14]) Let $(X, d)$ be a metric space, and let $F: X \rightarrow X$ be a map. A subset $S$ of $X$ is called a scrambled set of $F$ if for any two different points $x, y \in S$,

$$
\liminf _{n \rightarrow \infty} d\left(F^{n}(x), F^{n}(y)\right)=0, \quad \limsup _{n \rightarrow \infty} d\left(F^{n}(x), F^{n}(y)\right)>0 .
$$

The map $F$ is said to be chaotic in the Li-Yorke sense if there exists an uncountable scrambled set $S$ of $F$.

Definition 2 ([15]) A map $F: V \subset X \rightarrow V$ is said to be chaotic on $V$ in the sense of Devaney if

(i) $F$ is topologically transitive in $V$;

(ii) the periodic points of $F$ in $V$ are dense in $V$;

(iii) $F$ has sensitive dependence on initial conditions in $V$. 
By the result of [16], conditions (i) and (ii) imply (iii) if $F$ is continuous in $V$ that contains infinitely many points. Under some conditions, chaos in the sense of Devaney is stronger than that of Li-Yorke [17].

A nonperiodic boundary condition is given for Eq. (1) as

$$
x(n, k+1)=\varphi(x(n, p)), \quad n \geq 0,0 \leq p \leq k,
$$

where $p$ is an integer, and $\varphi: I \subset \mathbf{R} \rightarrow \mathbf{R}$ is a map. For any given initial condition $x(0, m)=$ $\phi(m), 0 \leq m \leq k+1$, where $\phi$ satisfies (6), Eq. (1) obviously has a unique solution satisfying this condition. By setting

$$
x_{n}=(x(n, 0), x(n, 1), \ldots, x(n, k))^{T} \in \mathbf{R}^{k+1}, \quad n \geq 0
$$

Equation (1) with (6) can be written as

$$
x_{n+1}=F\left(x_{n}\right), \quad n \geq 0,
$$

where

$$
F\left(x_{n}\right)=(f(x(n, 0), x(n, 1)), f(x(n, 1), x(n, 2)), \ldots, f(x(n, k), \varphi(x(n, p))))^{T} .
$$

System (7) is called the system induced by Eq. (1) with (6).

Definition 3 ([8]) Equation (1) with (6) is said to be chaotic in the sense of Devaney (or Li-Yorke) on $V \subset \mathbf{R}^{k+1}$ if its induced system (7) is chaotic in the sense of Devaney (or Li-Yorke) on $V$.

Definition 4 ([18]) Let $(X, d)$ be a metric space, and let $f: D \subset X \rightarrow X$ be a map. If there exist $m(\geq 2)$ subsets $V_{i}(1 \leq i \leq m)$ of $D$ with $V_{i} \cap V_{j}=\partial_{D} V_{i} \cap \partial_{D} V_{j}$ for each pair of $(i, j)$, $1 \leq i \neq j \leq m$, such that

$$
f\left(V_{i}\right) \supset \bigcup_{j=1}^{m} V_{j}, \quad 1 \leq i \leq m
$$

where $\partial_{D} V_{i}$ is the relative boundary of $V_{i}$ with respect to $D$, then $f$ is said to be a coupledexpanding map in $V_{i}, 1 \leq i \leq m$. Further, the map $f$ is said to be a strictly coupledexpanding map in $V_{i}, 1 \leq i \leq m$, if $d\left(V_{i}, V_{j}\right)>0$ for all $1 \leq i \neq j \leq m$.

Lemma 5 ([19]) Let $(X, d)$ be a metric space, and let $V_{j}(1 \leq j \leq m)$ be disjoint compact sets of $X$. If $f: D \equiv \bigcup_{j=1}^{m} V_{j} \rightarrow X$ is a strictly coupled-expanding continuous map in $V_{j}$, $1 \leq j \leq m$, then $f$ is chaotic in the sense of Li-Yorke.

Lemma $6([20,21])$ Let $(X, d)$ be a complete metric space, and let $f: D \subset X \rightarrow X$ be a map. Assume that there exist $k$ disjoint bounded closed subsets $V_{i}$ of $D, 1 \leq i \leq k$, such that $f$ is continuous in $\bigcup_{i=1}^{k} V_{i}$ and satisfies

(i) $f$ is strictly coupled-expanding in $V_{i}, 1 \leq i \leq k$; 
(ii) there exists a constant $\lambda>1$ such that

$$
d(f(x), f(y)) \geq \lambda d(x, y), \quad \forall x, y \in V_{i}, 1 \leq i \leq k .
$$

Then $f$ has an invariant Cantor set $V \subset \bigcup_{i=1}^{k} V_{i}$ such that $f: V \rightarrow V$ is topologically conjugate to the subshift $\Sigma_{k}^{+} \rightarrow \Sigma_{k}^{+}$. Consequently, $f$ is chaotic on $V$ in the Devaney and Li-Yorke senses.

\section{Main results}

In this section, we establish four chaotification schemes for Eq. (1) with tangent and cotangent functions.

Theorem 1 Consider the controlled system (2), that is,

$$
x(n+1, m)=f(x(n, m), x(n, m+1))+\varepsilon \tan (x(n, m)), \quad n \geq 0,0 \leq m \leq k<+\infty
$$

with (6). Suppose that

(i) there exist positive constants $r$ and $L$ such that

$$
\left|f\left(x_{1}, y_{1}\right)-f\left(x_{2}, y_{2}\right)\right| \leq L \max \left\{\left|x_{1}-x_{2}\right|,\left|y_{1}-y_{2}\right|\right\}, \quad \forall x_{1}, x_{2}, y_{1}, y_{2} \in[-r, r]
$$

(ii) $\varphi:[-r, r] \rightarrow[-r, r]$ is a map with $\varphi(0)=0$, and there exists a constant $\lambda>0$ such that

$$
|\varphi(x)-\varphi(y)| \leq \lambda|x-y|, \quad \forall x, y \in[-r, r]
$$

If $r>5 \pi / 4$, then for each constant $\varepsilon$ satisfying

$$
\varepsilon>\varepsilon_{0}:=\max \left\{\frac{5 \pi}{4}(1+L \max \{1, \lambda\})-f(0,0), \frac{\pi}{4}(1+5 L \max \{1, \lambda\})+f(0,0)\right\},
$$

there exists a Cantor set $\Lambda_{1} \subset\left[-\frac{\pi}{4}, \frac{\pi}{4}\right]^{k+1} \cup\left[\frac{3 \pi}{4}, \frac{5 \pi}{4}\right]^{k+1}$ such that system (2) with (6) is chaotic on $\Lambda_{1}$ in the Li-Yorke sense. Further, for each constant $\varepsilon$ satisfying

$$
\varepsilon>\max \left\{\varepsilon_{0}, 1+L \max \{1, \lambda\}\right\},
$$

there exists a Cantor set $\Lambda_{2} \subset\left[-\frac{\pi}{4}, \frac{\pi}{4}\right]^{k+1} \cup\left[\frac{3 \pi}{4}, \frac{5 \pi}{4}\right]^{k+1}$ such that system (2) with (6) is chaotic on $\Lambda_{2}$ in the Li-Yorke and Devaney senses.

Proof We use Lemmas 5 and 6. Let

$$
x_{n+1}=F\left(x_{n}\right)+\varepsilon \operatorname{Tan}\left(x_{n}\right):=G_{\varepsilon}\left(x_{n}\right), \quad n \geq 0,
$$

be the induced system of the controlled system (2) with (6), where $F\left(x_{n}\right)$ is (8), and

$$
\operatorname{Tan}\left(x_{n}\right)=(\tan (x(n, 0)), \tan (x(n, 1)), \ldots, \tan (x(n, k)))^{T}
$$


Let

$$
V_{1}=\left[-\frac{\pi}{4}, \frac{\pi}{4}\right]^{k+1}, \quad V_{2}=\left[\frac{3 \pi}{4}, \frac{5 \pi}{4}\right]^{k+1} .
$$

Then $V_{1}, V_{2} \subset[-r, r]^{k+1}$ are nonempty, closed, and bounded, and

$$
d\left(V_{1}, V_{2}\right)=\inf \left\{\|x-y\|: x \in V_{1}, y \in V_{2}\right\}=\frac{\pi}{2}>0 .
$$

The whole proof is divided into two parts.

Step 1 . System (2) with (6) is chaotic in the Li-Yorke sense.

By Lemma 5 we will show that $G_{\varepsilon}$ is a strictly coupled-expanding map in $V_{1}$ and $V_{2}$.

For each $x=(x(0), x(1), \ldots, x(k))^{T} \in V_{1}$ with $x(j)=-\pi / 4$, from (9) it follows that, for $0 \leq$ $j \leq k-1$,

$$
\begin{aligned}
G_{\varepsilon, j}(x) & =f(x(j), x(j+1))+\varepsilon \tan (x(j)) \\
& =f\left(-\frac{\pi}{4}, x(j+1)\right)+\varepsilon \tan \left(-\frac{\pi}{4}\right) \\
& \leq L \max \left\{\frac{\pi}{4},|x(j+1)|\right\}-\varepsilon+f(0,0) \\
& =\frac{\pi}{4} L-\varepsilon+f(0,0) \leq-\frac{\pi}{4},
\end{aligned}
$$

and for $j=k$, from (6), (9), and (10) it follows that

$$
\begin{aligned}
G_{\varepsilon, k}(x) & =f(x(k), \varphi(x(p)))+\varepsilon \tan (x(k)) \\
& =f\left(-\frac{\pi}{4}, \varphi(x(p))\right)+\varepsilon \tan \left(-\frac{\pi}{4}\right) \\
& \leq L \max \left\{\frac{\pi}{4},|\varphi(x(p))|\right\}-\varepsilon+f(0,0) \\
& \leq L \max \left\{\frac{\pi}{4}, \lambda|x(p)|\right\}-\varepsilon+f(0,0) \\
& \leq \frac{\pi}{4} L \max \{1, \lambda\}-\varepsilon+f(0,0) \leq-\frac{\pi}{4} .
\end{aligned}
$$

For each $x \in V_{1}$ with $x(j)=\pi / 4$, it follows from (6), (9), and (10) that, for $0 \leq j \leq k-1$,

$$
\begin{aligned}
G_{\varepsilon, j}(x) & =f\left(\frac{\pi}{4}, x(j+1)\right)+\varepsilon \tan \left(\frac{\pi}{4}\right) \\
& \geq-L \max \left\{\frac{\pi}{4},|x(j+1)|\right\}+\varepsilon+f(0,0) \\
& =-\frac{\pi}{4} L+\varepsilon+f(0,0) \geq \frac{5 \pi}{4},
\end{aligned}
$$


and for $j=k$,

$$
\begin{aligned}
G_{\varepsilon, k}(x) & =f\left(\frac{\pi}{4}, \varphi(x(p))\right)+\varepsilon \tan \left(\frac{\pi}{4}\right) \\
& \geq-L \max \left\{\frac{\pi}{4}, \lambda|x(p)|\right\}+\varepsilon+f(0,0) \\
& \geq-\frac{\pi}{4} L \max \{1, \lambda\}+\varepsilon+f(0,0) \geq \frac{5 \pi}{4} .
\end{aligned}
$$

By (9) and (10) $G_{\varepsilon}$ is continuous in $[-r, r]^{k+1}$. By the intermediate value theorem and (11)(14) we have $G_{\varepsilon}\left(V_{1}\right) \supset V_{1} \cup V_{2}$.

For each $x \in V_{2}$ with $x(j)=3 \pi / 4$, we have that for $0 \leq j \leq k-1$,

$$
\begin{aligned}
G_{\varepsilon, j}(x) & =f\left(\frac{3 \pi}{4}, x(j+1)\right)+\varepsilon \tan \left(\frac{3 \pi}{4}\right) \\
& \leq L \max \left\{\frac{3 \pi}{4},|x(j+1)|\right\}-\varepsilon+f(0,0) \\
& \leq \frac{5 \pi}{4} L-\varepsilon+f(0,0) \leq-\frac{\pi}{4},
\end{aligned}
$$

and for $j=k$,

$$
\begin{aligned}
G_{\varepsilon, k}(x) & =f\left(\frac{3 \pi}{4}, \varphi(x(p))\right)+\varepsilon \tan \left(\frac{3 \pi}{4}\right) \\
& \leq L \max \left\{\frac{3 \pi}{4}, \lambda|x(p)|\right\}-\varepsilon+f(0,0) \\
& \leq \frac{5 \pi}{4} L \max \{1, \lambda\}-\varepsilon+f(0,0) \leq-\frac{\pi}{4} .
\end{aligned}
$$

For each $x \in V_{2}$ with $x(j)=5 \pi / 4$, we have that for $0 \leq j \leq k-1$,

$$
\begin{aligned}
G_{\varepsilon, j}(x) & =f\left(\frac{5 \pi}{4}, x(j+1)\right)+\varepsilon \tan \left(\frac{5 \pi}{4}\right) \\
& \geq-L \max \left\{\frac{5 \pi}{4},|x(j+1)|\right\}+\varepsilon+f(0,0) \\
& =-\frac{5 \pi}{4} L+\varepsilon+f(0,0) \geq \frac{5 \pi}{4}
\end{aligned}
$$

and for $j=k$,

$$
\begin{aligned}
G_{\varepsilon, k}(x) & =f\left(\frac{5 \pi}{4}, \varphi(x(p))\right)+\varepsilon \tan \left(\frac{5 \pi}{4}\right) \\
& \geq-L \max \left\{\frac{5 \pi}{4}, \lambda|x(p)|\right\}+\varepsilon+f(0,0) \\
& \geq-\frac{5 \pi}{4} L \max \{1, \lambda\}+\varepsilon+f(0,0) \geq \frac{5 \pi}{4} .
\end{aligned}
$$

By the intermediate value theorem and (15)-(18) we have $G_{\varepsilon}\left(V_{2}\right) \supset V_{1} \cup V_{2}$. 
By the above discussion, $G_{\varepsilon}$ is a strictly coupled-expanding map in $V_{1}$ and $V_{2}$. Therefore by Lemma 5 system (2) with (6) is chaotic in the Li-Yorke sense.

Step 2. System (2) with (6) is chaotic in both Li-Yorke and Devaney senses.

Since $V_{1}, V_{2} \subset[-r, r]^{k+1}$, from (6), (9), and (10) it follows that for all $x, y \in V_{1}$ and $x, y \in$ $V_{2}$,

$$
\begin{aligned}
\|F(x)-F(y)\| & =\max \{|f(x(j), x(j+1))-f(y(j), y(j+1))|, 0 \leq j \leq k\} \\
& \leq L \max \{|x(j)-y(j)|,|\varphi(x(p))-\varphi(y(p))|, 0 \leq j, p \leq k\} \\
& \leq L \max \{|x(j)-y(j)|, \lambda|x(p)-y(p)|, 0 \leq j, p \leq k\} \\
& \leq L \max \{1, \lambda\}\|x-y\| .
\end{aligned}
$$

On the other hand, by Lagrange's mean value theorem, for all $x, y \in V_{1}$ and $x, y \in V_{2}$,

$$
\begin{aligned}
\|\operatorname{Tan}(x)-\operatorname{Tan}(y)\| & =\max \{|\tan (x(j))-\tan (y(j))|, 0 \leq j \leq k\} \\
& =\max \left\{\left|\sec ^{2} \xi(x(j)-y(j))\right|, 0 \leq j \leq k\right\} \\
& \geq\|x-y\|,
\end{aligned}
$$

where $\xi \in\left(-\frac{\pi}{4}, \frac{\pi}{4}\right) \cup\left(\frac{3 \pi}{4}, \frac{5 \pi}{4}\right)$. Hence from (19) and (20) it follows that for all $x, y \in V_{1}$ and $x, y \in V_{2}$,

$$
\left\|G_{\varepsilon}(x)-G_{\varepsilon}(y)\right\| \geq(\varepsilon-L \max \{1, \lambda\})\|x-y\| .
$$

Since $\varepsilon-L \max \{1, \lambda\}>1, G_{\varepsilon}$ satisfies assumption (ii) of Lemma 6 . Together with the result obtained in step 1, by Lemma 6 system (2) with (6) is chaotic in both Li-Yorke and Devaney senses. The proof is complete.

Theorem 2 Consider the controlled system (3), that is,

$$
x(n+1, m)=f(x(n, m), x(n, m+1))+\varepsilon \cot (x(n, m)), \quad n \geq 0,0 \leq m \leq k<+\infty
$$

with (6). Suppose that all the conditions in Theorem 1 hold. Then for all constants $\varepsilon, r$ satisfying

$$
\varepsilon>\max \left\{\frac{3 \pi}{4}(1+L \max \{1, \lambda\})-f(0,0), \frac{3 \pi}{4}(1+L \max \{1, \lambda\})+f(0,0)\right\}
$$

and $r>3 \pi / 4$, there exists a Cantor set $\Lambda \subset\left[-\frac{3 \pi}{4},-\frac{\pi}{4}\right]^{k+1} \cup\left[\frac{\pi}{4}, \frac{3 \pi}{4}\right]^{k+1}$ such that system (3) with (6) is chaotic on $\Lambda$ in both Li-Yorke and Devaney senses.

Proof We use Lemmas 5 and 6. The induced system of (3) with (6) is

$$
x_{n+1}=F\left(x_{n}\right)+\varepsilon \operatorname{Cot}\left(x_{n}\right):=H_{\varepsilon}\left(x_{n}\right), \quad n \geq 0,
$$

where $F$ is defined in (8), and

$$
\operatorname{Cot}\left(x_{n}\right)=(\cot (x(n, 0)), \cot (x(n, 1)), \ldots, \cot (x(n, k)))^{T}
$$


Let

$$
\tilde{V}_{1}=\left[-\frac{3 \pi}{4},-\frac{\pi}{4}\right]^{k+1}, \quad \widetilde{V}_{2}=\left[\frac{\pi}{4}, \frac{3 \pi}{4}\right]^{k+1} .
$$

Obviously, $\widetilde{V}_{1}, \widetilde{V}_{2} \subset[-r, r]^{k+1}$ are nonempty, closed, and bounded sets, and $d\left(\widetilde{V}_{1}, \widetilde{V}_{2}\right)=$ $\pi / 2>0$.

First, we show that $H_{\varepsilon}\left(\widetilde{V}_{i}\right) \supset \widetilde{V}_{1} \cup \widetilde{V}_{2}$ for $i=1,2$.

For each $x \in \widetilde{V}_{1}$ with $x(j)=-3 \pi / 4$, from (6), (9), and (10) it follows that for $0 \leq j \leq k-1$,

$$
\begin{aligned}
H_{\varepsilon, j}(x) & =f\left(-\frac{3 \pi}{4}, x(j+1)\right)+\varepsilon \cot \left(-\frac{3 \pi}{4}\right) \\
& \geq-L \max \left\{\frac{3 \pi}{4},|x(j+1)|\right\}+\varepsilon+f(0,0) \\
& =-\frac{3 \pi}{4} L+\varepsilon+f(0,0) \geq \frac{3 \pi}{4},
\end{aligned}
$$

and for $j=k$,

$$
\begin{aligned}
H_{\varepsilon, k}(x) & =f\left(-\frac{3 \pi}{4}, \varphi(x(p))\right)+\varepsilon \cot \left(-\frac{3 \pi}{4}\right) \\
& \geq-L \max \left\{\frac{3 \pi}{4}, \lambda|x(p)|\right\}+\varepsilon+f(0,0) \\
& =-\frac{3 \pi}{4} L \max \{1, \lambda\}+\varepsilon+f(0,0) \geq \frac{3 \pi}{4} .
\end{aligned}
$$

For each $x \in \widetilde{V}_{1}$ with $x(j)=-\pi / 4$, from (6), (9), and (10) it follows that for $0 \leq j \leq k-1$,

$$
\begin{aligned}
H_{\varepsilon, j}(x) & =f\left(-\frac{\pi}{4}, x(j+1)\right)+\varepsilon \cot \left(-\frac{\pi}{4}\right) \\
& \leq L \max \left\{\frac{\pi}{4},|x(j+1)|\right\}-\varepsilon+f(0,0) \\
& \leq \frac{3 \pi}{4} L-\varepsilon+f(0,0) \leq-\frac{3 \pi}{4}
\end{aligned}
$$

and for $j=k$,

$$
\begin{aligned}
H_{\varepsilon, k}(x) & =f\left(-\frac{\pi}{4}, \varphi(x(p))\right)+\varepsilon \cot \left(-\frac{\pi}{4}\right) \\
& \leq L \max \left\{\frac{\pi}{4}, \lambda|x(p)|\right\}-\varepsilon+f(0,0) \\
& \leq \frac{3 \pi}{4} L \max \{1, \lambda\}-\varepsilon+f(0,0) \leq-\frac{3 \pi}{4} .
\end{aligned}
$$


For each $x \in \widetilde{V}_{2}$ with $x(j)=\pi / 4$, for $0 \leq j \leq k-1$,

$$
\begin{aligned}
H_{\varepsilon, j}(x) & =f\left(\frac{\pi}{4}, x(j+1)\right)+\varepsilon \cot \left(\frac{\pi}{4}\right) \\
& \geq-L \max \left\{\frac{\pi}{4},|x(j+1)|\right\}+\varepsilon+f(0,0) \\
& \geq-\frac{3 \pi}{4} L+\varepsilon+f(0,0) \geq \frac{3 \pi}{4}
\end{aligned}
$$

and for $j=k$,

$$
\begin{aligned}
H_{\varepsilon, k}(x) & =f\left(\frac{\pi}{4}, \varphi(x(p))\right)+\varepsilon \cot \left(\frac{\pi}{4}\right) \\
& \geq-L \max \left\{\frac{\pi}{4}, \lambda|x(p)|\right\}+\varepsilon+f(0,0) \\
& \geq-\frac{3 \pi}{4} L \max \{1, \lambda\}+\varepsilon+f(0,0) \geq \frac{3 \pi}{4} .
\end{aligned}
$$

For each $x \in \widetilde{V}_{2}$ with $x(j)=3 \pi / 4$, for $0 \leq j \leq k-1$,

$$
\begin{aligned}
H_{\varepsilon, j}(x) & =f\left(\frac{3 \pi}{4}, x(j+1)\right)+\varepsilon \cot \left(\frac{3 \pi}{4}\right) \\
& \leq L \max \left\{\frac{3 \pi}{4},|x(j+1)|\right\}-\varepsilon+f(0,0) \\
& =\frac{3 \pi}{4} L-\varepsilon+f(0,0) \leq-\frac{3 \pi}{4}
\end{aligned}
$$

and for $j=k$,

$$
\begin{aligned}
H_{\varepsilon, k}(x) & =f\left(\frac{3 \pi}{4}, \varphi(x(p))\right)+\varepsilon \cot \left(\frac{3 \pi}{4}\right) \\
& \leq L \max \left\{\frac{3 \pi}{4}, \lambda|x(p)|\right\}-\varepsilon+f(0,0) \\
& \leq \frac{3 \pi}{4} L \max \{1, \lambda\}-\varepsilon+f(0,0) \leq-\frac{3 \pi}{4} .
\end{aligned}
$$

By the intermediate value theorem and (21)-(28), we have $H_{\varepsilon}\left(\widetilde{V}_{i}\right) \supset \widetilde{V}_{1} \cup \widetilde{V}_{2}, i=1$, 2 . So by Lemma 5 system (3) with (6) is chaotic in the Li-Yorke sense.

Next, we show that $H_{\varepsilon}$ satisfies assumption (ii) in Lemma 6.

By Lagrange's mean value theorem we can verify that for all $x, y \in \widetilde{V}_{1}$ and $x, y \in \widetilde{V}_{2}$,

$$
\begin{aligned}
\|\operatorname{Cot}(x)-\operatorname{Cot}(y)\| & =\max \{|\cot (x(j))-\cot (y(j))|, 0 \leq j \leq k\} \\
& =\max \left\{\left|-\csc ^{2} \theta(x(j)-y(j))\right|, 0 \leq j \leq k\right\} \\
& \geq\|x-y\|,
\end{aligned}
$$

where $\theta \in\left(-\frac{3 \pi}{4},-\frac{\pi}{4}\right) \cup\left(\frac{\pi}{4}, \frac{3 \pi}{4}\right)$. Hence, by (19), for all $x, y \in \widetilde{V}_{1}$ and $x, y \in \widetilde{V}_{2}$,

$$
\left\|H_{\varepsilon}(x)-H_{\varepsilon}(y)\right\| \geq(\varepsilon-L \max \{1, \lambda\})\|x-y\| .
$$


Since $\varepsilon>\frac{3}{4} \pi(1+L \max \{1, \lambda\})$, we have $\varepsilon-L \max \{1, \lambda\}>1$. Thus $H_{\varepsilon}$ satisfies assumption (ii) in Lemma 6. By Lemma 6 system (3) with (6) is chaotic in both Li-Yorke and Devaney senses. This completes the proof.

Now we consider the controlled systems (4) and (5). For convenience, we give a periodic boundary condition for Eq. (1):

$$
x(n, k+1)=x(n, 0), \quad n \geq 0 .
$$

We have the following two results.

Theorem 3 Consider the controlled system (4), that is,

$$
x(n+1, m)=f(x(n, m), x(n, m+1))+\varepsilon \tan (x(n, m+1)), \quad n \geq 0,0 \leq m \leq k<+\infty,
$$

with (29). Suppose that condition (i) in Theorem 1 holds. Then all the results in Theorem 1 hold for system (4) with (29), except that $\max \{1, \lambda\}$ in Theorem 1 is replaced by 1.

Proof The induced system of (4) with (29) can be written as

$$
x_{n+1}=\widetilde{F}\left(x_{n}\right)+\varepsilon \operatorname{Tan}\left(\widehat{x}_{n}\right):=\widetilde{G}_{\varepsilon}\left(x_{n}\right), \quad n \geq 0,
$$

where

$$
\begin{aligned}
& \widetilde{F}\left(x_{n}\right)=(f(x(n, 0), x(n, 1)), f(x(n, 1), x(n, 2)), \ldots, f(x(n, k), x(n, 0)))^{T}, \\
& \operatorname{Tan}\left(\widehat{x}_{n}\right)=(\tan (x(n, 1)), \tan (x(n, 2)), \ldots, \tan (x(n, k)), \tan (x(n, 0)))^{T} .
\end{aligned}
$$

Let $V_{1}$ and $V_{2}$ be the same as in Theorem 1. We divide the proof into two parts.

Step 1. System (4) with (29) is chaotic in the Li-Yorke sense.

For each $x \in V_{1}$ with $x(j+1)=-\pi / 4$, from (9) it follows that for $0 \leq j \leq k-1$,

$$
\begin{aligned}
\widetilde{G}_{\varepsilon, j}(x) & =f(x(j), x(j+1))+\varepsilon \tan (x(j+1)) \\
& =f\left(x(j),-\frac{\pi}{4}\right)+\varepsilon \tan \left(-\frac{\pi}{4}\right) \\
& \leq L \max \left\{|x(j)|, \frac{\pi}{4}\right\}-\varepsilon+f(0,0) \\
& =\frac{\pi}{4} L-\varepsilon+f(0,0) \leq-\frac{\pi}{4},
\end{aligned}
$$

and for $j=k$, from (9) and (29) it follows that $x(k+1)=x(0)=-\pi / 4$, so that

$$
\begin{aligned}
\widetilde{G}_{\varepsilon, k}(x) & =f(x(k), x(0))+\varepsilon \tan (x(0)) \\
& =f\left(x(k),-\frac{\pi}{4}\right)+\varepsilon \tan \left(-\frac{\pi}{4}\right) \\
& \leq L \max \left\{|x(k)|, \frac{\pi}{4}\right\}-\varepsilon+f(0,0) \\
& =\frac{\pi}{4} L-\varepsilon+f(0,0) \leq-\frac{\pi}{4} .
\end{aligned}
$$


For each $x \in V_{1}$ with $x(j+1)=\pi / 4$, by (9) and (29), $x(k+1)=x(0)=\pi / 4$ for $j=k$. Therefore for $0 \leq j \leq k$,

$$
\begin{aligned}
\widetilde{G}_{\varepsilon, j}(x) & =f\left(x(j), \frac{\pi}{4}\right)+\varepsilon \tan \left(\frac{\pi}{4}\right) \\
& \geq-L \max \left\{|x(j)|, \frac{\pi}{4}\right\}+\varepsilon+f(0,0) \\
& =-\frac{\pi}{4} L+\varepsilon+f(0,0) \geq \frac{5 \pi}{4} .
\end{aligned}
$$

For each $x \in V_{2}$ with $x(j+1)=3 \pi / 4,0 \leq j \leq k$, from (9) and (29) it follows that

$$
\begin{aligned}
\widetilde{G}_{\varepsilon, j}(x) & =f\left(x(j), \frac{3 \pi}{4}\right)+\varepsilon \tan \left(\frac{3 \pi}{4}\right) \\
& \leq L \max \left\{|x(j)|, \frac{3 \pi}{4}\right\}-\varepsilon+f(0,0) \\
& \leq \frac{5 \pi}{4} L-\varepsilon+f(0,0) \leq-\frac{\pi}{4},
\end{aligned}
$$

and for each $x \in V_{2}$ with $x(j+1)=5 \pi / 4,0 \leq j \leq k$, we have

$$
\begin{aligned}
\widetilde{G}_{\varepsilon, j}(x) & =f\left(x(j), \frac{5 \pi}{4}\right)+\varepsilon \tan \left(\frac{5 \pi}{4}\right) \\
& \geq-L \max \left\{|x(j)|, \frac{5 \pi}{4}\right\}+\varepsilon+f(0,0) \\
& =-\frac{5 \pi}{4} L+\varepsilon+f(0,0) \geq \frac{5 \pi}{4} .
\end{aligned}
$$

By the intermediate value theorem and (31)-(35) we have $\widetilde{G}_{\varepsilon}\left(V_{i}\right) \supset V_{1} \cup V_{2}, i=1,2$. Therefore by Lemma 5 system (4) with (29) is chaotic in the Li-Yorke sense.

Step 2. System (4) with (29) is chaotic in both Li-Yorke and Devaney senses.

Since $V_{1}, V_{2} \subset[-r, r]^{k+1}$, from (9) and (29) it follows that for all $x, y \in V_{1}$ and $x, y \in V_{2}$,

$$
\begin{aligned}
\|\widetilde{F}(x)-\widetilde{F}(y)\| & =\max \{|f(x(j), x(j+1))-f(y(j), y(j+1))|, 0 \leq j \leq k\} \\
& \leq L \max \{|x(j)-y(j)|, 0 \leq j \leq k\} \\
& =L\|x-y\| .
\end{aligned}
$$

On the other hand, by Lagrange's mean value theorem, for all $x, y \in V_{1}$ and $x, y \in V_{2}$,

$$
\begin{aligned}
\|\operatorname{Tan}(\widehat{x})-\operatorname{Tan}(\widehat{y})\| & =\max \{|\tan (x(j))-\tan (y(j))|, 0 \leq j \leq k\} \\
& =\max \left\{\left|\sec ^{2} \eta(x(j)-y(j))\right|, 0 \leq j \leq k\right\} \\
& \geq\|x-y\|,
\end{aligned}
$$

where $\eta \in\left(-\frac{\pi}{4}, \frac{\pi}{4}\right) \cup\left(\frac{3 \pi}{4}, \frac{5 \pi}{4}\right)$. Hence from (36) and (37) it follows that

$$
\left\|\widetilde{G}_{\varepsilon}(x)-\widetilde{G}_{\varepsilon}(y)\right\| \geq(\varepsilon-L)\|x-y\|, \quad \forall x, y \in V_{1} \text { or } x, y \in V_{2} .
$$


Since $\varepsilon-L>1, \widetilde{G}_{\varepsilon}$ satisfies assumption (ii) of Lemma 6 . Together with the result obtained in step 1, by Lemma 6 system (4) with (29) is chaotic in both Li-Yorke and Devaney senses. This completes the proof.

Remark 1 The boundary conditions imposed on systems (2)-(3) and (4)-(5) are different. If (6) is imposed on system (4), then in (32), $x(k+1)=\varphi(x(p))=-\pi / 4,0 \leq p \leq k$, but we cannot ensure that $x(p) \in\left[-\frac{\pi}{4}, \frac{\pi}{4}\right]$. Thus $x \in V_{1}$ may not hold. Therefore (29) is imposed on systems (4) and (5).

Theorem 4 Consider the controlled system (5), that is,

$$
x(n+1, m)=f(x(n, m), x(n, m+1))+\varepsilon \cot (x(n, m+1)), \quad n \geq 0,0 \leq m \leq k<+\infty,
$$

with (29). Suppose that condition (i) in Theorem 1 holds. Then all the results in Theorem 2 hold for system (5) with (29), where $\max \{1, \lambda\}=1$.

Proof We use Lemmas 5 and 6. Let

$$
x_{n+1}=\widetilde{F}\left(x_{n}\right)+\varepsilon \operatorname{Cot}\left(\widehat{x}_{n}\right):=\widetilde{H}_{\varepsilon}\left(x_{n}\right), \quad n \geq 0,
$$

be the induced system of system (5) with (29), where $\widetilde{F}$ is defined in (30), and

$$
\operatorname{Cot}\left(\widehat{x}_{n}\right)=\left(\cot (x(n, 1)), \cot (x(n, 2)), \ldots, \cot (x(n, k), \cot (x(n, 0)))^{T}\right.
$$

Let $\widetilde{V}_{1}$ and $\widetilde{V}_{2}$ be the same as in Theorem 2 .

For each $x \in \widetilde{V}_{1}$ with $x(j+1)=-3 \pi / 4,0 \leq j \leq k$, from (9) and (29) it follows that

$$
\begin{aligned}
\widetilde{H}_{\varepsilon, j}(x) & =f\left(x(j),-\frac{3 \pi}{4}\right)+\varepsilon \cot \left(-\frac{3 \pi}{4}\right) \\
& \geq-L \max \left\{|x(j)|, \frac{3 \pi}{4}\right\}+\varepsilon+f(0,0) \\
& =-\frac{3 \pi}{4} L+\varepsilon+f(0,0) \geq \frac{3 \pi}{4},
\end{aligned}
$$

and for each $x \in \widetilde{V}_{1}$ with $x(j+1)=-\pi / 4,0 \leq j \leq k$, from (9) and (29) it follows that

$$
\begin{aligned}
\widetilde{H}_{\varepsilon, j}(x) & =f\left(x(j),-\frac{\pi}{4}\right)+\varepsilon \cot \left(-\frac{\pi}{4}\right) \\
& \leq L \max \left\{|x(j)|, \frac{\pi}{4}\right\}-\varepsilon+f(0,0) \\
& \leq \frac{3 \pi}{4} L-\varepsilon+f(0,0) \leq-\frac{3 \pi}{4} .
\end{aligned}
$$

By the intermediate value theorem and (38)-(39) we have $\widetilde{H}_{\varepsilon}\left(\widetilde{V}_{1}\right) \supset \widetilde{V}_{1} \cup \widetilde{V}_{2}$. Similarly, we can prove that $\widetilde{H}_{\varepsilon}\left(\widetilde{V}_{2}\right) \supset \widetilde{V}_{1} \cup \widetilde{V}_{2}$. 
By Lagrange's mean value theorem we can verify that for all $x, y \in \widetilde{V}_{1}$ and $x, y \in \widetilde{V}_{2}$,

$$
\begin{aligned}
\|\operatorname{Cot}(\widehat{x})-\operatorname{Cot}(\widehat{y})\| & =\max \{|\cot (x(j))-\cot (y(j))|, 0 \leq j \leq k\} \\
& =\max \left\{\left|-\csc ^{2} \theta(x(j)-y(j))\right|, 0 \leq j \leq k\right\} \\
& \geq\|x-y\|,
\end{aligned}
$$

where $\theta \in\left(-\frac{3 \pi}{4},-\frac{\pi}{4}\right) \cup\left(\frac{\pi}{4}, \frac{3 \pi}{4}\right)$. Together with (36), for all $x, y \in \widetilde{V}_{1}$ and $x, y \in \widetilde{V}_{2}$, we have

$$
\left\|\widetilde{H}_{\varepsilon}(x)-\widetilde{H}_{\varepsilon}(y)\right\| \geq(\varepsilon-L)\|x-y\|
$$

where $\varepsilon>\frac{3 \pi}{4}(1+L)>1+L$. By Lemma 6 system (5) with (29) is chaotic in both Li-Yorke and Devaney senses. This completes the proof.

\section{Examples}

In this section, we discuss three examples with computer simulations.

Example 1 Consider the controlled system (2) with (6), where

$$
f(x, y)= \begin{cases}\frac{1}{32} x y+\frac{1}{2} \pi, & x, y \in[-4,4] \\ \frac{1}{2}|x y|, & \text { else }\end{cases}
$$

and

$$
\varphi(x)=\frac{1}{2} x, \quad \forall x \in \mathbf{R} .
$$

It is evident that $\left|f_{x}(x, y)\right|+\left|f_{y}(x, y)\right| \leq 1 / 4$ for all $x, y \in[-4,4]$, that is,

$$
\left|f\left(x_{1}, y_{1}\right)-f\left(x_{2}, y_{2}\right)\right| \leq \frac{1}{4} \max \left\{\left|x_{1}-x_{2}\right|,\left|y_{1}-y_{2}\right|\right\}, \quad \forall x_{1}, x_{2}, y_{1}, y_{2} \in[-4,4] .
$$

Thus $f$ and $\varphi$ satisfy all the assumptions in Theorem 1 with $r=4, L=1 / 4, \lambda=1 / 2$, and $f(0,0)=\pi / 2$. By Theorem 1 , for any $\varepsilon>17 \pi / 16$, there exists a Cantor set $\Lambda \subset$ $\left[-\frac{1}{4} \pi, \frac{1}{4} \pi\right]^{k+1} \cup\left[\frac{3}{4} \pi, \frac{5}{4} \pi\right]^{k+1}$ such that the controlled system is chaotic on $\Lambda$ in both LiYorke and Devaney senses. Two simulation results on two-dimensional plane $(x(\cdot, 0)$, $x(\cdot, 1))$ and three-dimensional space $(x(\cdot, 0), x(\cdot, 1), x(\cdot, 2))$ are given in Fig. 1 for $p=1$, $k=1,2$, and $\varepsilon=9 \pi / 8$, which exhibit complicated dynamical behaviors of the controlled system on $\Lambda$.

Example 2 Consider the controlled system (3) with (6), where

$$
f(x, y)= \begin{cases}\frac{1}{9} x^{2}+\frac{1}{3} y, & x, y \in[-3,3] \\ \cos (x+y), & \text { else }\end{cases}
$$

and

$$
\varphi(x)=\frac{4}{3} x, \quad \forall x \in \mathbf{R} .
$$



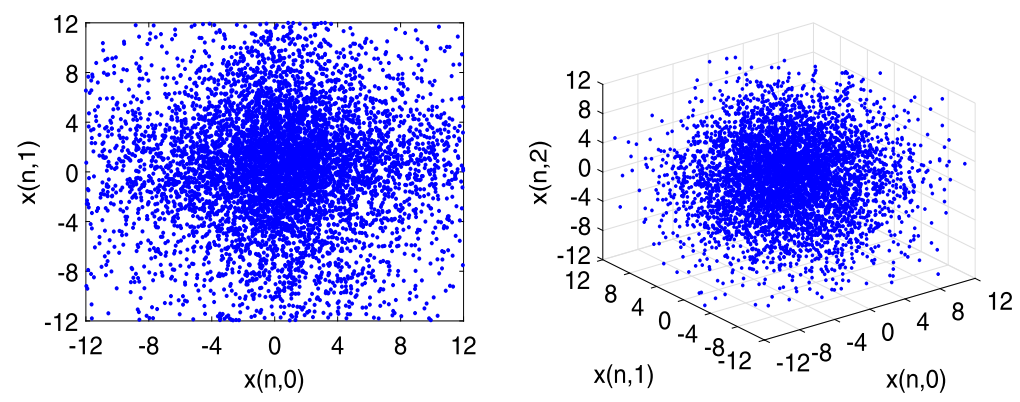

Figure 1 Simulations for system (2) with (6), where $n=0,1, \ldots, 10,000$ and $p=0$. In the 2-D graph, the initial values are taken as $x(0,0)=0.1$ and $x(0,1)=-0.1$. The initial values are $x(0,0)=0.1, x(0,1)=-0.1$, and $x(0,2)=0.1$ in the $3-D$ graph
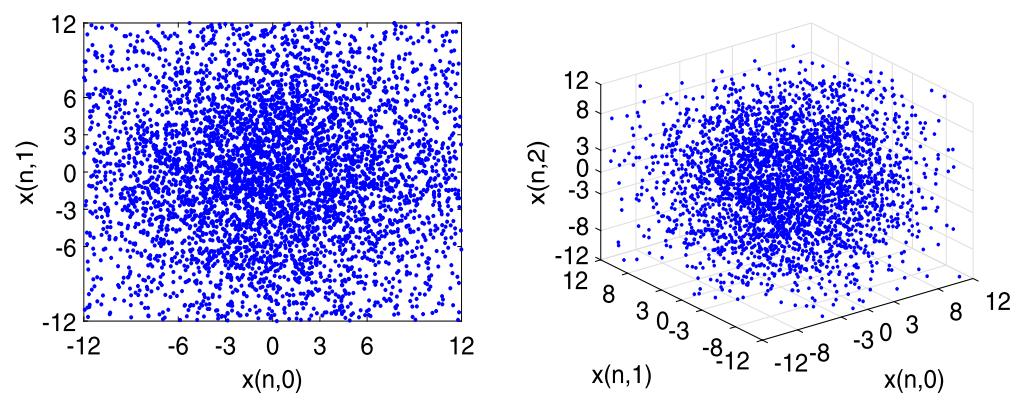

Figure 2 Simulations for system (3) with (6), where $n=0,1, \ldots, 10,000$ and $p=1$. In the 2-D graph, $k=1$, and the initial values are $x(0,0)=1, x(0,1)=-0.1$. In the 3 -D graph, $k=2$, and the initial value are $x(0,0)=0.1$, $x(0,1)=-0.1$, and $x(0,2)=0.1$
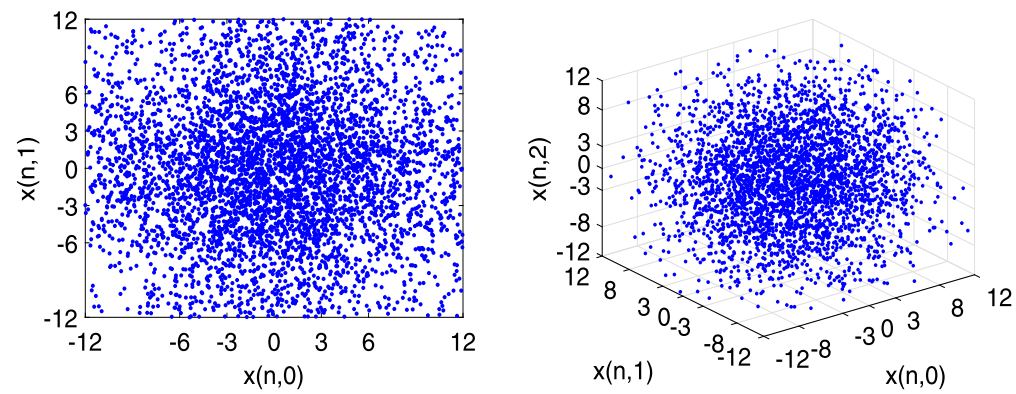

Figure 3 Simulations for system (5) with (29), where $n=0,1, \ldots, 10,000$. In the 2-D graph, $k=1$, and the initial values are $x(0,0)=1, x(0,1)=-0.1$. In the 3-D graph, $k=2$, and the initial values are $x(0,0)=0.1, x(0,1)=-0.1$, and $x(0,2)=0.1$

Obviously, $f(0,0)=0$ and $\left|f_{x}(x, y)\right|+\left|f_{y}(x, y)\right| \leq 1$ for all $x, y \in[-3,3]$, which implies that

$$
\left|f\left(x_{1}, y_{1}\right)-f\left(x_{2}, y_{2}\right)\right| \leq \max \left\{\left|x_{1}-x_{2}\right|,\left|y_{1}-y_{2}\right|\right\}, \quad \forall x_{1}, x_{2}, y_{1}, y_{2} \in[-3,3] .
$$

Hence $f$ and $\varphi$ satisfy all the assumptions in Theorem 2 with $r=3, L=1, \lambda=4 / 3$. Thus, by Theorem 2 , for any constant $\varepsilon>7 \pi / 4$, there exists a Cantor set $\Lambda \subset\left[-\frac{3}{4} \pi,-\frac{1}{4} \pi\right]^{k+1} \cup$ $\left[\frac{1}{4} \pi, \frac{3}{4} \pi\right]^{k+1}$ such that the controlled system (3) with (6) is chaotic on $\Lambda$ in both Li-Yorke 
and Devaney senses. Two simulation results are shown in Fig. 2 for $p=1$ and $\varepsilon=2 \pi$, which indicate that the controlled system has very complicated dynamical behaviors on $\Lambda$.

Example 3 Consider the controlled system (5) with (29), where $f(x, y)$ is (40). By the previous discussion, $f$ satisfies all the assumptions in Theorem 4 with $r=3$ and $L=1$. Thus, by Theorem 4 , for any constant $\varepsilon>3 \pi / 2$, there exists a Cantor set $\Lambda \subset\left[-\frac{3}{4} \pi,-\frac{1}{4} \pi\right]^{k+1} \cup$ $\left[\frac{1}{4} \pi, \frac{3}{4} \pi\right]^{k+1}$ such that the controlled system is chaotic on $\Lambda$ in both Li-Yorke and Devaney senses. Simulation results are shown in Fig. 3 for $\varepsilon=2 \pi$, which show that the controlled system has very complicated dynamical behaviors on $\Lambda$.

\section{Acknowledgements}

Not applicable.

Funding

Not applicable

Availability of data and materials

Not applicable.

\section{Competing interests}

The authors declare that they have no competing interests.

\section{Authors' contributions}

WL contributed to the idea of this paper, wrote the manuscript, and revised it. HG proved the theorems and wrote this paper. Both authors read and approved the final manuscript.

\section{Publisher's Note}

Springer Nature remains neutral with regard to jurisdictional claims in published maps and institutional affiliations.

Received: 13 August 2020 Accepted: 6 December 2020 Published online: 04 January 2021

\footnotetext{
References

1. Gang, H., Qu, Z.: Controlling spatiotemporal chaos in coupled map lattice systems. Phys. Rev. Lett. 72(1), 68-71 (1994)

2. Willeboordse, F.: The spatial logistic map as a simple prototype for spatiotemporal chaos. Chaos 13(2), 533-540 (2003)

3. Wiggins, S., Mazel, D.: Introduction to applied nonlinear dynamical systems and chaos. Comput. Phys. 4 (1998)

4. Chen, G., Liu, S.: On spatial periodic orbits and spatial chaos. Int. J. Bifurc. Chaos 13(04), 935-941 (2003)

5. Chen, G., Tian, C., Shi, Y: Stability and chaos in 2-D discrete systems. Chaos Solitons Fractals 25(3), 637-647 (2005)

6. Shi, Y: Chaos in first-order partial difference equations. J. Differ. Equ. Appl. 14(2), 109-126 (2008)

7. Liang, W., Shi, Y., Zhang, C.: Chaotification for a class of first-order partial difference equations. Int. J. Bifurc. Chaos 14(2), 717-733 (2008)

8. Shi, Y., Yu, P., Chen, G.: Chaotification of discrete dynamical system in Banach spaces. Int. J. Bifurc. Chaos 16(09), 2615-2636 (2006)

9. Liang, W., Guo, H.: Chaotification of first-order partial difference equations. Int. J. Bifurc. Chaos 30(15), 2050229 (2020)

10. Liang, W., Zhang, Z.: Chaotification schemes of first-order partial difference equations via sine functions. J. Differ. Equ. Appl. 25, 665-675 (2019)

11. Liang, W., Zhang, Z.: Anti-control of chaos for first-order partial difference equations via sine and cosine functions. Int. J. Bifurc. Chaos 29(10), 1950140 (2019)

12. Chen, G., Lai, D.: Feedback anticontrol of discrete chaos. Int. J. Bifurc. Chaos 8(07), 1585-1590 (1998)

13. Wang, X., Chen, G.: Chaotification via arbitrarily small feedback controls: theory, method, and applications. Int. J. Bifurc. Chaos 10(03), 549-570 (2000)

14. Li, T., Yorke, J.: Period three implies chaos. Am. Math. Mon. 82, 985-992 (1975)

15. Devaney, R.: An Introduction to Chaotic Dynamical Systems, 2nd edn. Addison-Wesley, Reading (1989)

16. Banks, J., Brooks, J., Cairns, G.: On Devaney's definition of chaos. Am. Math. Mon. 99(4), 332-334 (1992)

17. Huang, W., Ye, X.: Devaney's chaos or 2-scattering implies Li-Yorke's chaos. Topol. Appl. 117(3), 259-272 (2002)

18. Shi, Y., Yu, P.: Chaos induced by regular snap-back repellers. J. Math. Anal. Appl. 337(2), 1480-1494 (2008)

19. Zhang, X., Shi, Y., Chen, G.: Constructing chaotic polynomial maps. Int. J. Bifurc. Chaos 19(02), 531-543 (2009)

20. Shi, Y., Ju, H., Chen, G.: Coupled-expanding maps and one-sided symbolic dynamical systems. Chaos Solitons Fractals 39(5), 2138-2149 (2009)

21. Shi, Y., Xing, Q.: Dense distribution of chaotic maps in continuous map spaces. Dyn. Stab. Syst. 26(4), 519-535 (2011)
} 